\title{
3D numerical simulation of particle-fluid flow in open channels with a porous bed
}

\author{
J. Zhang, P. Dupont, M. Hellou \& B. Benmezroua \\ Laboratoire LGCGM, INSA de Rennes, \\ Université Européenne de Bretagne, France
}

\begin{abstract}
A 3D CFD (Computational Fluid Dynamics)-DEM (Discrete Element Method) method is used for simulation of complicated behaviour including interaction between particle and fluid in the hybrid domain, involving both a porous region and a clear fluid region. A comparison of the results taken from our numerical tool with the experimental data available in two literatures proved that the numerical tool developed here can be used in situations where the Reynolds number and the porosity are different. The effects of the dynamic characteristics of porous medium, including angle of inclination, inlet flow velocity, discharge velocity and porosity, on the particle movement were further investigated. The statistical results demonstrate that the average horizontal component of the particle velocity follows a Gaussian distribution for each case. The average particle velocity and the average flow velocity within the free-fluid region increase with increasing angle of inclination, inlet flow velocity, discharge velocity and porosity. This 3D CFD-DEM method, as demonstrated by the scheme proposed and applied in this work, may serve as an effective simulation tool for the simulation of particle-fluid flows.
\end{abstract}

Keywords: particle-fluid flow, open channels, porous media, free surface flow.

\section{Introduction}

Transport phenomena at the interface between a fluid and porous medium has remained an important topic of research because of their fundamental interest in understanding the phenomena of infiltration into the porous medium and its wide field of applications in industry and environmental analyses, for example, river hydraulics, urban hydrology, pollution and remediation of soils, water treatment, 
etc. Flows over a porous medium are combined the flows both over and through the porous medium. Because of the interaction between surface flow and seepage flow inside porous region, this interaction affects both the flow discharge and the sediment discharge.

The effect of the interaction between the seepage flow and the surface flow has been studied by several forerunners. Beavers and Joseph [1] they found an empirical relationship for slip velocity at the interface; Rudraiah [2], Poulikakos and Kazmierczak [3], Vafai and Thiyagaraja [4], and Vafai and Kim [5] proposed the continuity conditions at the interface for both velocity and shear stresses. Sahraoui and Kaviany [6] and Ochoa-Tapia and Whitaker [7, 8] have used macroscopic approaches to study the velocity near the interface and also developed the interface shear stress jump condition, but this approach just show the complexities of the interface area. And Choi and Waller [9] applied a macroscopic single domain approach to treat complex interaction of the flow near the interfacial region.

Some studies have presented findings about the effect of turbulent flow above and through porous bed. Chu and Gelhar [10] presented analytical results to explain the reason why organized fluctuation appears due to non-Darcy law as a resistance law for the flow in a highly permeable porous medium. Zippe and Graf [11] presented the boundary resistance of the permeable surface is higher than that of the impermeable boundary with the same roughness. Shimizu et al. [12] based on experimental findings, concluded a mathematical model of the velocities within the porous medium under turbulent flow, However, the model was based on a function of the slip interfacial velocity, which is not known a priori. Thus, applying such a model is rather difficult. Mendoza and Zhou [13] and Zhou and Mendoza [14] presented the turbulent flow characteristics over a porous bed and the velocity distribution in surface flow, however, the details of transport phenomena near the boundary of the permeable interface was not investigated.

Either a microscopic or macroscopic model can be used in the numerical investigation of the turbulent flow over the porous region. Most of the studies on porous medium flow apply macroscopic models within a two-dimensional (2D) porous bed (e.g., [15-25]). Vafai et al. [26] found a synthesis of models for turbulent transport through porous media. Pedras and de Lemos [23] presented a macroscopic turbulent model which was a $k-\varepsilon$ model based on the formulations developed from the numerical results, described the characteristics of the flow turbulent kinetic energy. Chan et al. [27] presented the numerical solutions for turbulent 2D flow in a channel with a porous medium, they applied a singledomain approach and concluded that the level of turbulent penetration depended strongly on the damping effect of the porous medium itself. However using the macroscopic models could not have detailed insight into the flow structure, due to the development of the computational capacity, a complex computational fluid dynamics (CFD) could be performed. Prinos et al. [28] presented microscopic computation of flow over and within a porous bed. They also provided very valuable measured data. 
In the present study, the characteristics of a free surface flow over a porous bed in a three-dimensional (3D) inclined open channel are studied numerically using the microscopic approach. The flow is described by the Navier-stokes equations, while the rigid spherical particles which assembled the porous medium are described by the Discrete Element Method (DEM). The equations were solved using the commercial software CCFD (2007) and PFC3D 4.0 (2008).

\section{Mathematical equations}

Figure 1 shows the flow investigated here. The rectangular channel is partially filled with a layer of a porous material. A free surface flow passes longitudinally from left to right. At the bottom is an impermeable wall.

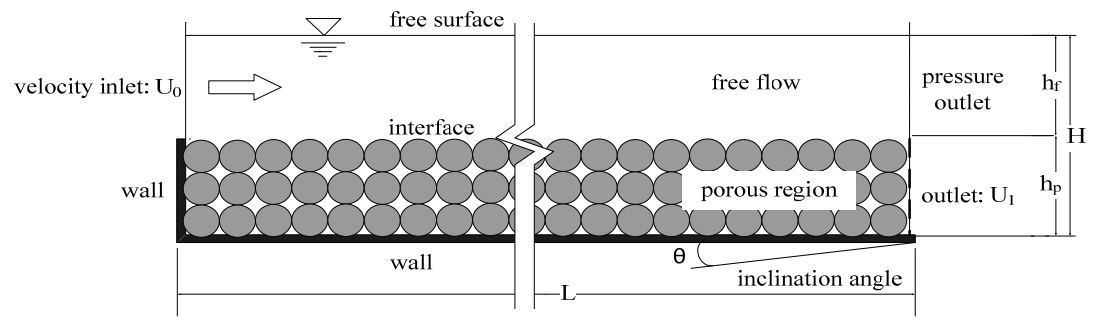

Figure 1: Sketch of computational domain with boundary conditions in a 2D view.

The flow in the whole flow region is calculated from the continuity and the Navier-Stokes equations with a porosity term and an additional body force term to account for the presence of particles in the fluid, which are given by

$$
\begin{gathered}
\frac{\partial \phi}{\partial t}+\nabla \cdot(\phi \mathbf{v})=0 \\
\rho_{\mathrm{f}} \frac{\partial \phi \mathbf{v}}{\partial t}+\rho_{\mathrm{f}} \boldsymbol{v} \cdot \nabla(\phi \mathbf{v})=-\phi \nabla p+\mu_{\mathrm{f}} \nabla^{2}(\phi \mathbf{v})+\mathbf{f}_{\mathrm{b}}
\end{gathered}
$$

where $\rho_{\mathrm{f}}$ is the density of the fluid, $\phi$ is porosity, $v$ is the fluid velocity, $p$ is the fluid pressure, $\mu_{\mathrm{f}}$ is the dynamic viscosity of the fluid and $\mathbf{f}_{\mathrm{b}}$ is a body force per unit volume. The porosity and body force in these fluid elements are determined by the DEM.

The equations of motion for particles with an additional forcing term to account for interaction with the fluid are expressed as:

$$
\begin{gathered}
m \frac{\partial \mathbf{u}}{\partial t}=\mathbf{f}_{\text {mech }}+\mathbf{f}_{\text {fluid }}+m \mathbf{g} \\
\frac{\partial \boldsymbol{\omega}}{\partial t}=\frac{\mathbf{M}}{I}
\end{gathered}
$$


where $\mathbf{U}$ is the particle velocity, $m$ is the particle mass, $\mathbf{f}_{\text {fluid }}$ is the total force applied by the fluid on the particle, $\mathbf{f}_{\text {mech }}$ is the sum of additional forces acting on the particle, $\mathbf{g}$ is the gravitational acceleration, $\boldsymbol{\omega}$ is the particle angular velocity, $I$ is the moment of inertia and $\mathbf{M}$ is the moment acting on the particle.

$\mathbf{f}_{\text {fluid }}$ is made up of two parts: the drag force and a force due to the fluid pressure gradient:

$$
\begin{gathered}
\mathbf{f}_{\text {fluid }}=\mathbf{f}_{\text {drag }}+\frac{4}{3} \pi r^{3}\left(\nabla p-\rho_{\mathrm{f}} \mathbf{g}\right) \\
\mathbf{f}_{\text {drag }}=\mathbf{f}_{0} \phi^{-\chi}
\end{gathered}
$$

where $\mathbf{f}_{0}$ is the force on a single particle. The $\phi^{-\chi}$ term is an empirical factor to account for the local porosity. This correction term makes the force applicable to both fixed and fluidized porosity systems and for a large range of Reynolds numbers $[29,30]$.

The single particle force in an infinite medium is defined as:

$$
\mathbf{f}_{0}=\left(\frac{1}{2} C_{\mathrm{d}} \rho_{\mathrm{f}} \pi r^{2}|\mathbf{u}-\mathbf{v}|(\mathbf{u}-\mathbf{v})\right)
$$

where $r$ is the particle radius, $C_{d}$ is a drag coefficient, has been given by Dallavalle [31]:

$$
C_{d}=\left(0.63+\frac{4.8}{\sqrt{R e_{\mathrm{p}}}}\right)^{2}
$$

The particle Reynolds number, $R e_{\mathrm{p}}$ is:

$$
R e_{\mathrm{p}}=\frac{2 \rho_{\mathrm{f}} r|\mathbf{u}-\mathbf{v}|}{\mu_{\mathrm{f}}}
$$

The empirical coefficient $\chi$ is defined as:

$$
\chi=3.7-0.65 \exp \left[-\frac{\left(1.5-\log _{10}\left(R e_{\mathrm{p}}\right)\right)^{2}}{2}\right]
$$

The body force per unit volume, $\mathbf{f}_{\mathrm{b}}$, in each fluid element is determined as:

$$
\mathbf{f}_{\mathrm{b}}=\frac{\sum_{j} \mathbf{f}_{\text {drag }}^{j}}{V}
$$

where $V$ is the volume of the fluid element.

DEM and CFD two-way coupling is numerically achieved as follows. Eqns (1) and (2) are solved with the CFD code CCFD, and eqns (3)-(11) are solved with the DEM code PFC3D. The porosity and the body force are determined in PFC3D and divided by volume in CCFD. The fluid velocity and 
fluid pressure in every element are determined by CCFD and send to PFC3D in each coupling data exchange.

The standard $k-\varepsilon$ model is used in turbulent flow regime. The volume of fluid method (VOF) has been in usage for fluid flow calculations. The polyhedron method is used for calculating the porosity in a fluid element, this method represents a particle as a cube with height, length and width equal to the diameter of the particle, and it has the advantage that the change in porosity is smooth as a particle moves from one fluid element to another.

\section{Validation test of the method}

The best way to examine and increase the understanding of the numerical simulation discussed above yield is to compare it with the existing experimental and the simulation data. Accordingly, the computational conditions, including inlet flow velocity, the depth of free surface flow, thickness and porosity of porous regions, position of interface and inclination angle $\theta$ of the channel were set to match the conditions of the available reference data.

Table 1: $\quad$ Measured experimental parameters from Shimizu et al. [12] and Prinos et al. [28].

\begin{tabular}{|c|c|c|c|c|c|c|c|}
\hline Case & $\phi$ & $\begin{array}{c}H \\
(\mathrm{~m})\end{array}$ & $\begin{array}{c}h_{\mathrm{f}} \\
(\mathrm{m})\end{array}$ & $h_{\mathrm{f}} / H$ & $\theta$ & $\begin{array}{c}U_{\mathrm{f}} \\
(\mathrm{m} / \mathrm{s})\end{array}$ & $R e$ \\
\hline $1 \mathrm{a}$ & 0.3800 & 0.1458 & 0.0567 & 0.3889 & $0.1^{\circ}$ & 0.435 & $2.351 \times 10^{4}$ \\
\hline $1 \mathrm{~b}$ & 0.3800 & 0.1752 & 0.0861 & 0.4914 & $0.1^{\circ}$ & 0.487 & $3.869 \times 10^{4}$ \\
\hline $1 \mathrm{c}$ & 0.3800 & 0.1781 & 0.0591 & 0.3318 & $0.1^{\circ}$ & 0.417 & $2.339 \times 10^{4}$ \\
\hline 2a & 0.8286 & 0.0850 & 0.0300 & 0.3529 & $0.1^{\circ}$ & 0.253 & $7.581 \times 10^{3}$ \\
\hline 2b & 0.8286 & 0.1050 & 0.0500 & 0.4762 & $0.1^{\circ}$ & 0.287 & $1.437 \times 10^{4}$ \\
\hline
\end{tabular}

Case 1: Shimizu et al. [12] performed experiments modelling of flow in a laboratory flume ( $8 \mathrm{~m}$ long, $0.21 \mathrm{~m}$ wide) partially filled with glass beads (with diameter, $D=2.97 \mathrm{~cm}$ ). The glass beads were arrayed as the porous medium. The geometric and hydrodynamic characteristics are shown in Figure 2 case 1 and Table 1 (1a, $1 \mathrm{~b}$ and 1c), respectively.

Figure 3 compares the computed velocity distributions $U_{\mathrm{s}}$ below the fluid/porous interface with the experimental data obtained by Shimizu et al. [12]. Notably, the present numerical model accurately represents the general trend of the velocity distributions. The interface velocities are quite high, with values varying from 0.17 to 0.26 , which are much higher than the respective laminar flow values over a porous medium, as predicted by Choi and Waller [9].

Case 2: Prinos et al. [28] investigated fully developed and uniform flow in a flume with a porous bed. The flume was $12 \mathrm{~m}$ long, $0.25 \mathrm{~m}$ wide and $0.5 \mathrm{~m}$ high. The porous bed was simulated using spatially periodic rods $(D=10 \mathrm{~mm})$ in a non-staggered arrangement. The geometric and hydrodynamic characteristics are shown in Figure 2 (case 2) and Table 1 ( $2 \mathrm{a}$ and $2 \mathrm{~b}$ ), respectively. 


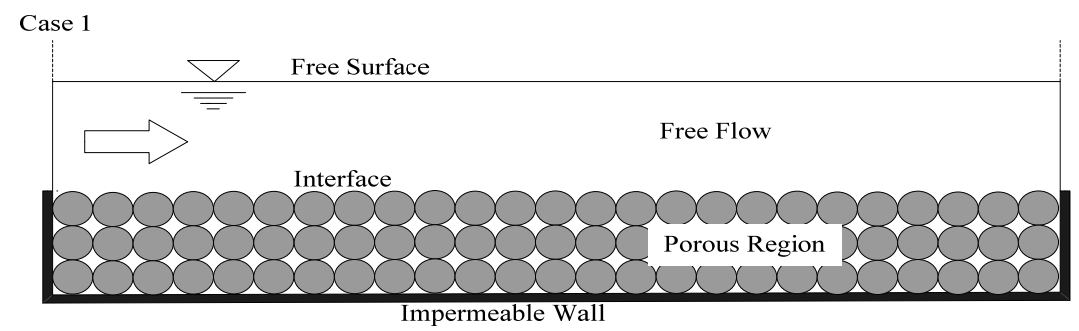

Side view of Shimmizu et al. [12] experiments.

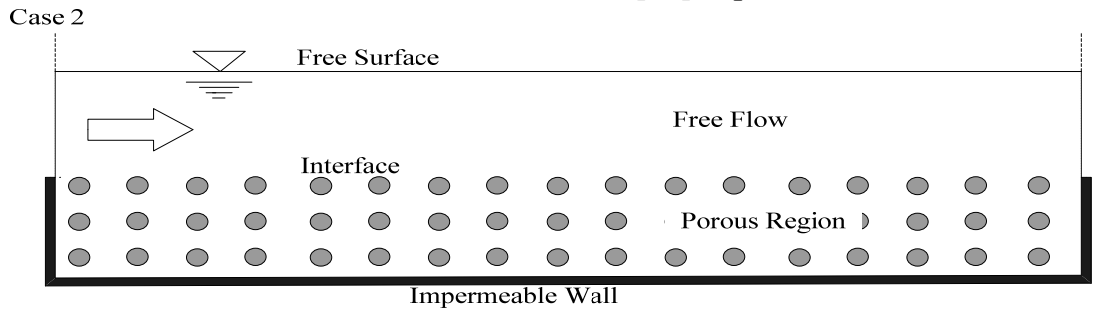

Side view of Prinos et al. [28] experiments.

Figure 2: Schematic diagram of the model tests.

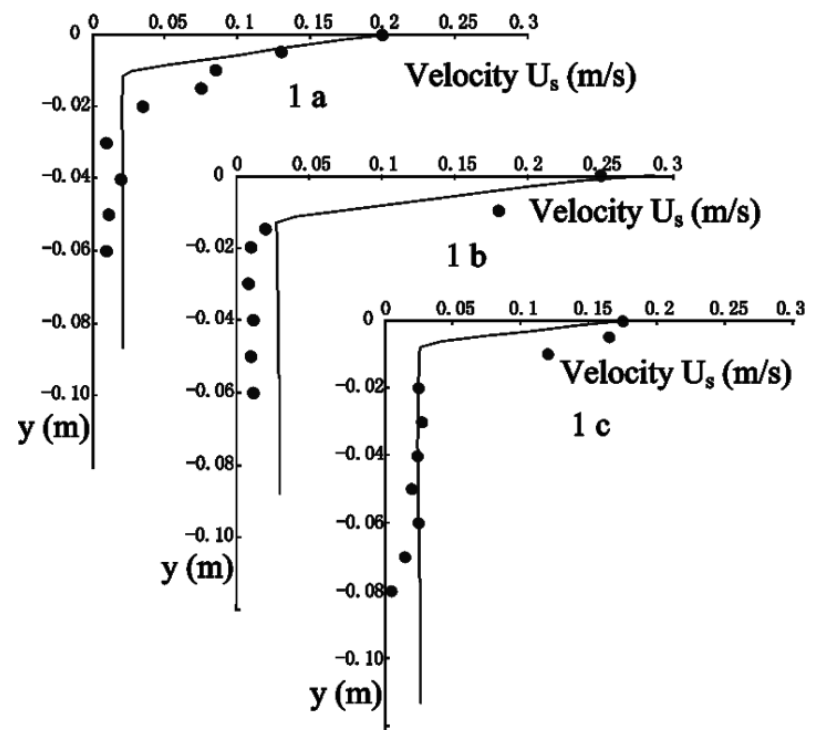

Figure 3: Comparison of velocity distribution within porous medium. Lines, computations; symbols, measurements (Shimizu et al. [12]).

Figure 4 plots the turbulent velocities above the fluid/porous interface in both the cases, as obtained by using the present model. The velocities are presented in wall coordinates $y^{+}\left(=y U_{*} / v\right)$ and $U^{+}\left(=U / U_{*}\right)$ to enable them to be directly 
compared with both the measured and computed data by Prinos et al. [28]. The logarithmic law in the fully turbulent region, $U^{+}=(1 / \kappa) \ln y^{+}+C(\kappa=0.41, C=$ 5.29), for flow over a smooth impermeable bed are also plotted. The presence of the porous medium causes a significant reduction of the velocities in the clear fluid region, compared to the flow over the smooth impermeable bed.

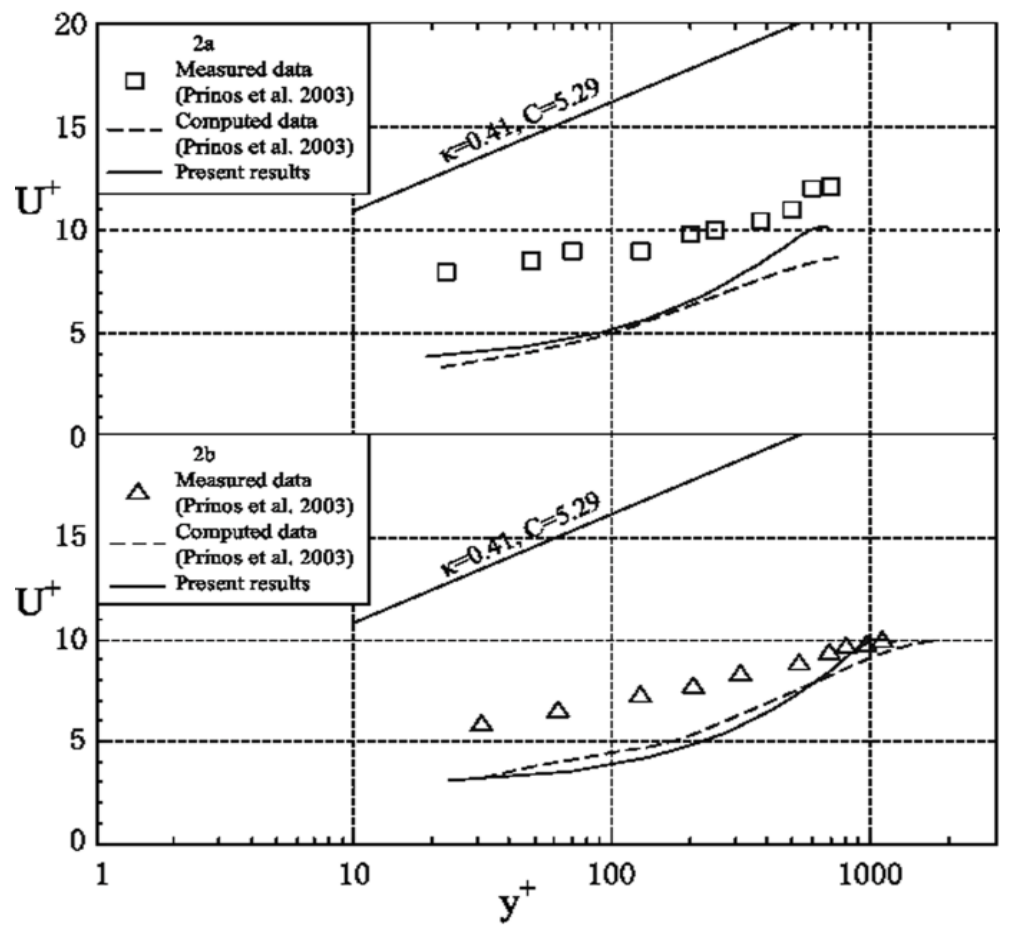

Figure 4: Turbulent velocity distribution over porous region in wall coordinates. Lines, computations; symbols, measurements (Prinos et al. [28]).

This is due to the penetration of the turbulence and the momentum exchange near the fluid/porous interface. Similar trends are observed in the measured velocity distribution, but it indicates higher velocities than computed ones, this may be caused by limited length of the porous region in the laboratory channel (Prinos et al. [28]).

\section{Solid particle transport over the porous bed}

After the above validation, the numerical methods are used to simulate the motion of one ball transport over the porous medium by the flow. The computational domain having dimensions equal to $0.03 \mathrm{~m} \times 0.015 \mathrm{~m} \times 0.20 \mathrm{~m}$, a grid consisting of $90 \times 45 \times 600$ points for the computation domain. The samples 
are from 20 particles which are added to the flow field one by one, a particle is deleted if it reaches the edge of the area of interest, in the meantime, the next particle is added in the middle of the flow field over the surface of porous media, without initial velocity. During which the system is statistically steady. The average velocity of a particle over a distance along the channel of $60 \mathrm{~mm}$ is calculated. Particle has a diameter of $2 \mathrm{~mm}$ and density of $2500 \mathrm{~kg} / \mathrm{m}^{3}$, friction coefficient of 0.4 , stiffness coefficient of $1 \times 10^{6} \mathrm{~N} / \mathrm{m}$, and the critical damping ratio is 0.04 . Fixed solid particles with the same values of material parameters are used to assemble the porous media. The thickness of porous medium is $6 \mathrm{~mm}$ and the inlet flow thickness is $5 \mathrm{~mm}$. Table 2 summarizes the parameters used in the sensitivity analysis.

Table 2: $\quad$ Parameters used in the sensitivity analysis.

\begin{tabular}{|c|c|c|c|c|c|}
\hline Case & $\phi$ & $\theta$ & $\begin{array}{c}U_{0} \\
(\mathrm{~m} / \mathrm{s})\end{array}$ & $\begin{array}{c}U_{1} \\
(\mathrm{~m} / \mathrm{s})\end{array}$ & $R e$ \\
\hline case1 & 0.3955 & $0.1^{\circ}$ & 0.30 & 0 & $1.174 \times 10^{3}$ \\
\hline case2 & 0.3955 & $0.6^{\circ}$ & 0.30 & 0 & $1.158 \times 10^{3}$ \\
\hline case3 & 0.3955 & $1.1^{\circ}$ & 0.30 & 0 & $1.152 \times 10^{3}$ \\
\hline case4 & 0.3955 & $0.1^{\circ}$ & 0.40 & 0 & $1.667 \times 10^{3}$ \\
\hline case5 & 0.3955 & $0.1^{\circ}$ & 0.45 & 0 & $1.891 \times 10^{3}$ \\
\hline case6 & 0.3955 & $0.1^{\circ}$ & 0.30 & 0.03 & $1.178 \times 10^{3}$ \\
\hline case7 & 0.3955 & $0.1^{\circ}$ & 0.30 & 0.15 & $1.150 \times 10^{3}$ \\
\hline case8 & 0.2595 & $0.1^{\circ}$ & 0.30 & 0 & $1.191 \times 10^{3}$ \\
\hline case9 & 0.4764 & $0.1^{\circ}$ & 0.30 & 0 & $1.151 \times 10^{3}$ \\
\hline
\end{tabular}

The analytical results show that the velocity distributions of particles in different cases (Figures 5, 6, 7 and 8) follow a Gaussian distribution with a high correlation coefficient (Table 3), which can be expressed into the following correlation:

$$
P\left(U_{\mathrm{px}}\right)=\frac{A}{\sigma \sqrt{2 \pi}} \exp \left(-\frac{\left(U_{\mathrm{px}}-\mu\right)^{2}}{2 \sigma^{2}}\right)
$$

where $P$ represents the probability distribution of horizontal component of the particle velocity.

Figure 5(a) presents the inclination angle influence on the distributions of average horizontal component of the particle velocity. A greater inclination angle $\theta$ corresponds to faster average particle speed. Figure 5(b) presents the corresponding variation of the computed velocity profiles. Evidently, the greater inclination angle causes a higher flow around the fluid/porous interface and within free-fluid region, and the higher flow velocity near the interface together with down slope component of gravity causes the increase in particle velocity.

Figure 6(a) presents the inlet flow velocity influence on the distributions of average horizontal component of the particle velocity. The average particle 
Table 3: $\quad$ Fitting parameters of Gaussian distribution.

\begin{tabular}{|c|c|c|c|c|}
\hline Case & $A$ & $\mu$ & $\sigma$ & $R^{2}$ \\
\hline case1 & 0.09175 & 0.08217 & 0.00332 & 0.94944 \\
\hline case2 & 0.12866 & 0.12944 & 0.00692 & 0.88813 \\
\hline case3 & 0.11386 & 0.16879 & 0.00500 & 0.91239 \\
\hline case4 & 0.37106 & 0.13737 & 0.01801 & 0.91444 \\
\hline case5 & 0.86015 & 0.19708 & 0.04015 & 0.85127 \\
\hline case6 & 0.10043 & 0.09841 & 0.00485 & 0.96700 \\
\hline case7 & 0.05912 & 0.10980 & 0.00322 & 0.94139 \\
\hline case8 & 0.05724 & 0.06761 & 0.00292 & 0.85000 \\
\hline case9 & 0.09584 & 0.08732 & 0.00524 & 0.92470 \\
\hline
\end{tabular}

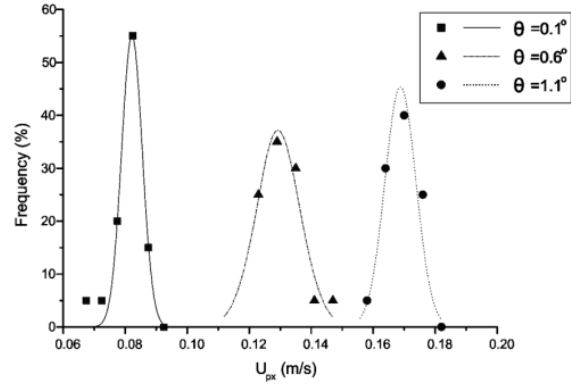

(a)

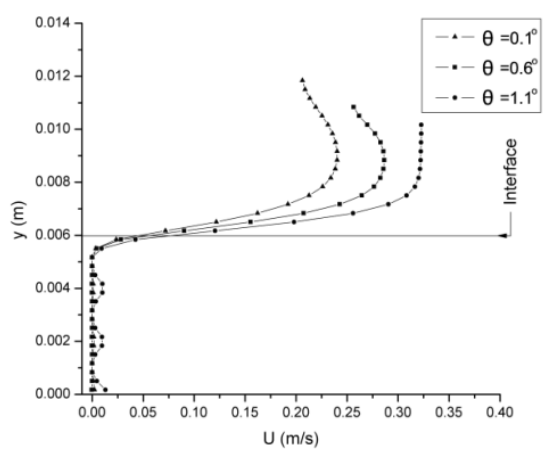

(b)

Figure 5: (a) Distributions of average horizontal component of the particle velocity for different inclination angle, lines correspond to Gaussian distribution. (b) Flow velocity profiles above and within the porous region for different inclination angle.

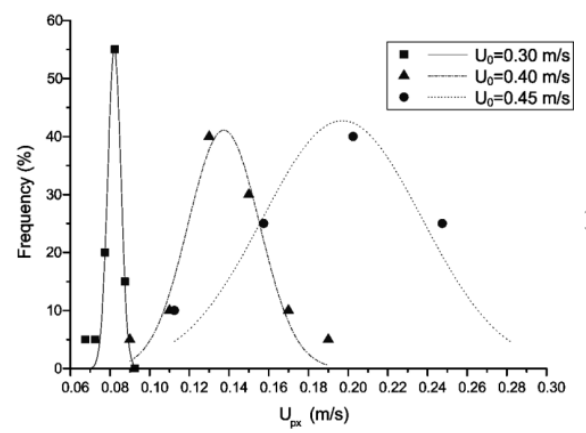

(a)

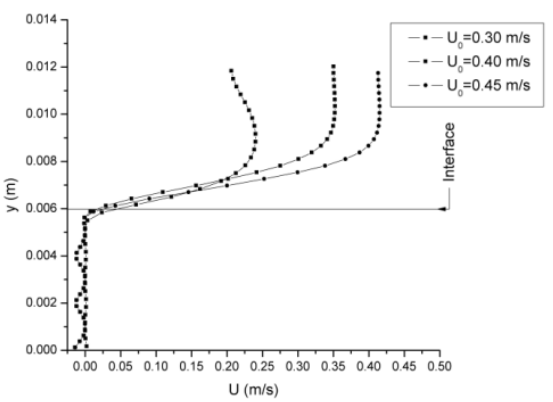

(b)

Figure 6: (a) Distributions of average horizontal component of the particle velocity for different inlet flow velocity, lines correspond to Gaussian distribution. (b) Flow velocity profiles above and within the porous region for different inlet flow velocity. 
velocity significantly increases with increasing of the inlet flow velocity. Figure 6(b) shows flow velocity profile above and within the porous region for different inlet flow velocity.

Figure 7(a) investigates the discharge velocity influence on the distributions of average horizontal component of the particle velocity. The average particle velocity significantly increases when there is a discharge rate through the porous media; however, the effect is not so obvious at a higher discharge velocity. The velocity profiles in Figure 7(b) show the similar trends, and the fluid height slightly decreases with increasing discharge velocity.

Figure 8(a) presents the effect of porosity $\phi$ on the distributions of average horizontal component of the particle velocity. When the porosity of porous

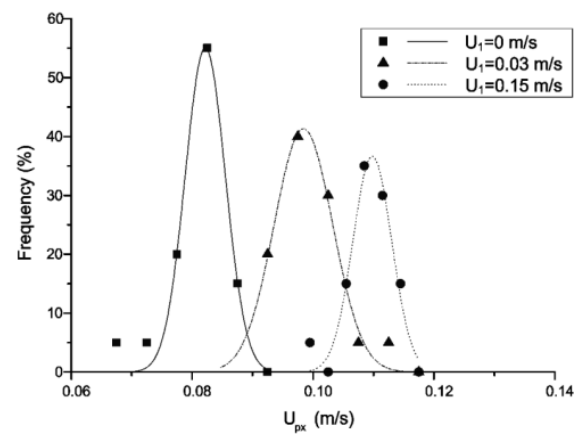

(a)

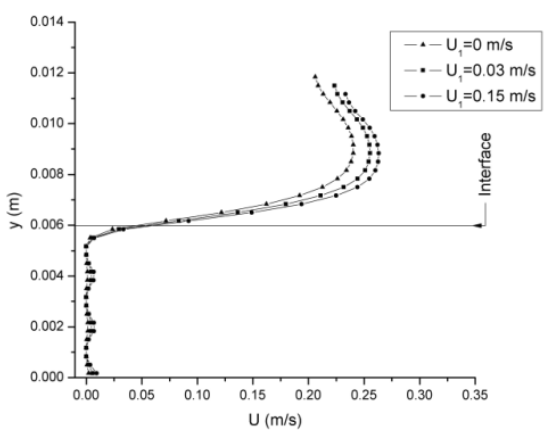

(b)

Figure 7: (a) Distributions of average horizontal component of the particle velocity for different discharge velocity, lines correspond to Gaussian distribution. (b) Flow velocity profiles above and within the porous region for different discharge velocity.

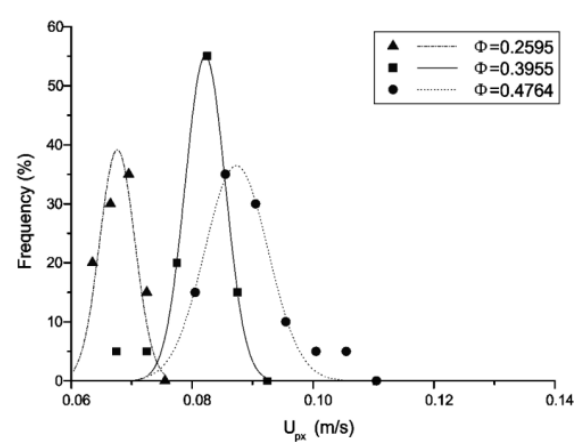

(a)

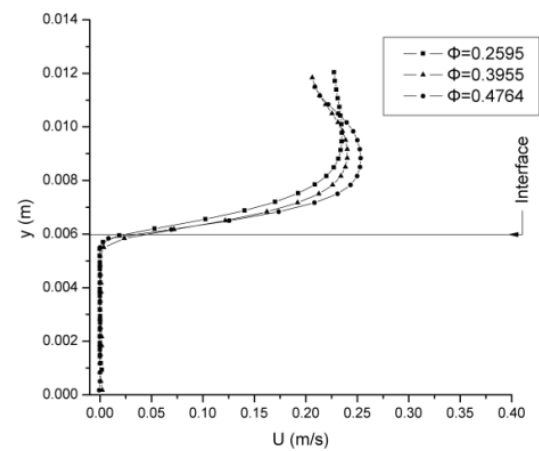

(b)

Figure 8: (a) Distributions of average horizontal component of the particle velocity for different porosity, lines correspond to Gaussian distribution. (b) Flow velocity profiles above and within the porous region for different porosity. 
media is bigger, the average particle velocity is higher. Figure 8 (b) shows the greater porosity causes a higher flow within free-fluid region, but things change near the free surface, the flow over the porous medium with the lowest porosity have highest velocity at the free surface, while the fluid height slightly decreases with increasing porosity.

\section{References}

[1] Beavers, G.S., Joseph, D.D., Boundary conditions at a naturally permeable wall. J. Fluid Mech., 30, pp. 197-207, 1967.

[2] Rudraiah, N., Coupled parallel Rows in a channel and a bounding porous medium of finite thickness. J. Fluids Engrg., 107, pp. 322-329, 1985.

[3] Poulikakos, D., \& Kazmierczak, M., Forced convection in a duct partially filled with a porous material. J. Heat Transfer, 109, pp. 653-662, 1987.

[4] Vafai, K., \& Thiyagaraja, R., Analysis of flow and heat transfer at the interface region of a porous medium. Int. J. Heat Mass Transfer, 30, pp. 1391-1405, 1987.

[5] Vafai, K., \& Kim, S.J., Forced convection in a channel filled with a porous medium: an exact solution. J. Heat Transfer, 111, pp. 1103-1106, 1989.

[6] Sahraoui, M., \& Kaviany, M., Slip and no-slip velocity boundary conditions at interface of porous, plain media: conduction. Int. J. Heat Mass Transfer, 35, pp. 927-943, 1992.

[7] Ochoa-Tapia, A.J., \& Whitaker, S., Momentum transfer at the boundary between a porous medium and a homogeneous fluid. I: Theoretical development. Int. J. Heat Mass Transfer, 38(14), pp. 2635-2646, 1995.

[8] Ochoa-Tapia, A.J., \& Whitaker, S., Momentum transfer at the boundary between a porous medium and a homogeneous fluid. II: Comparison with experiment. Int. J. Heat Mass Transfer, 38(14), pp. 2647-2655, 1995.

[9] Choi, C.Y., \& Waller, P.M., Momentum transport mechanism for water flow over porous media. J. Environ. Engrg., 123 (8), pp. 792-799, 1997.

[10] Chu, Y.H., \& Gelhar, L.W., Turbulent pipe flow with granular permeable boundaries. Report No. 148, Ralph M. Parsons Laboratory for Water Resources and Hydrodynamics. Dept. of Civil Engineering, Massachusetts Institute of Technology, Cambridge, Mass., 1972.

[11] Zippe, H.J., \& Graf, W.H., Turbulent boundary-layer flow over permeable and non-permeable rough surfaces. J. Hydraul. Res., 21 (1), pp. 51-65, 1983.

[12] Shimizu, Y., Tsujimoto, T., Nakagawa, H., Experimental and macroscopic modeling of flow in highly porous medium under free surface flow. $J$. Hydrosci. Hydraul. Engrg., 8 (1), pp. 69-78, 1990.

[13] Mendoza, C., \& Zhou, D., Effect of porous bed on turbulent stream flow above bed. J. Hydraul. Engrg., 118 (9), pp. 1222-1240, 1992.

[14] Zhou, D., \& Mendoza, C., Flow through porous bed of turbulent stream. $J$. Engrg. Mech., 119 (2), pp. 365-383, 1993.

[15] Wang, H., \& Takle, E.S., Boundary-layer flow and turbulence near porous obstacles. Boundary-Lay. Meteorol., 74, pp. 73-88, 1995. 
[16] Travkin, V.S., \& Catton, I., A two temperature model for turbulent flow and heat transfer in a porous layer. J. Fluids Engrg., 117, pp. 181-188, 1995.

[17] Masuoka, T., \& Takatsu, Y., Turbulence model for flow through porous media. Int. J. Heat Mass Transfer, 39, pp. 2803-2809, 1996.

[18] Antohe, B.V., \& Lage, J.L., A general two-equation macroscopic turbulence model for incompressible flow in porous media. Int. J. Heat Mass Transfer, 40, pp. 3013-3024. 1997.

[19] Kuwahara, F., Kameyama, Y., Yamashita, S., Nakayama, A., Numerical modeling of turbulent flow in porous media using a spatially periodic array. J. Porous Media, 1, pp. 47-55, 1998.

[20] Takatsu, Y., \& Masuoka, T., Turbulent phenomena in flow through porous media. J. Porous Media, 3, pp. 243-251, 1998.

[21] Nakayama, A., \& Kuwahara, F., A macroscopic turbulence model for flow in a porous medium. J. Fluids Engrg. 121, pp. 427-433. 1999.

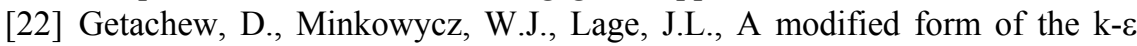
model for turbulent flow of an incompressible fluid in porous media. Int. $J$. Heat Mass Transfer, 43, pp. 2909-2915, 2000.

[23] Pedras, M.H.J., \& de Lemos, M.J.S., On the definition of turbulent kinetic energy for flow in porous media. Int. Commun. Heat Mass Transfer, 27 (2), pp. 211-220, 2000.

[24] Pedras, M.H.J., \& de Lemos, M.J.S., Macroscopic turbulence modeling for incompressible flow through undeformable porous media. Int. J. Heat Mass Transfer, 44 (6), pp. 1081-1093, 2001.

[25] Pedras, M.H.J., \& de Lemos, M.J.S., Simulation of turbulent flow in porous media using a spatially periodic array and a low Re two equation closure. Numer. Heat Transfer Part A, 39 (1), pp. 35-59, 2001.

[26] Vafai, K., Khanafer, K., Minkowycz, W.J., Bejan, A., Synthesis of models for turbulent transport through porous media. Handbook of Numerical Heat Transfer, Wiley, New York, 2005.

[27] Chan H.C., Huang W.C., Leu J.M., Lai C.J., Macroscopic modeling of turbulent flow over a porous medium. Int. J. Heat and Fluid Flow, 28, pp. 1157-1166, 2007.

[28] Prinos, P., Sofialidis, D., Keramaris, E., Turbulent flow over and within a porous bed. J. Hydraul. Engrg. 129 (9), pp. 720-733, 2003.

[29] Di Felice, R., The voidage function for fluid-particle interaction systems. Int. J. Multiphase Flow, 20, pp. 153-159, 1994.

[30] Xu, B.H., \& Yu, A.B., Numerical simulation of the gas-solid flow in a fluidized bed by combining discrete particle method with computational fluid dynamics. Chem. Engrg. Sci, 52, pp. 2786-2809, 1997.

[31] Dallavalle, J.M., Micromeritics: the Technology of Fine Particles, 2nd edn., Pitman, London, pp. 14-40, 1948. 